\title{
Land management and biodiversity maintenance: a case study in grasslands in the Coastal Plain of Rio Grande do Sul
}

\author{
Camila Leal Bonilha, Bianca Ott Andrade, Mariana de Souza Vieira, Pedro Joel Silva da Silva Filho, \\ Rosângela Gonçalves Rolim, Gerhard Ernst Overbeck \& Ilsi lob Boldrini.
}

\begin{abstract}
Universidade Federal do Rio Grande do Sul, Laboratório de Estudos em Vegetação Campestre, Av. Bento Gonçalves, 9500 Bloco IV Prédio 43432, Sala 109, Bairro Agronomia, CEP 91501-970, Porto Alegre, RS, Brazil. camila.bonilha431@gmail.com, andradebo@gmail.com marianasvbio@gmail.com, pedrojssf@gmail.com, rosangelagrolim@yahoo.com.br, gerhard.overbeck@ufrgs.br, ilsi.boldrini@ufrgs.br
\end{abstract}

Recebido em 10.VI.2015

Aceito em 28.VII. 2017

DOI $10.21826 / 2446-8231201772206$

ABSTRACT - Coastal grasslands are one of the most threatened types of grasslands in the southern Brazil. To evaluate if areas with different landuse history differ in current grassland species composition and structure, four areas were selected according to current management: Entisols areas used for extensive livestock breeding (GR1 and GR2 sites); Alfisols areas used for rice production with fallow periods (GRC), and for rotation with soybean (GSB). In each area, soil and grassland vegetation were characterized. We verified that GR1, GSB, GRC and GR2 sites followed a decreasing order of species richness. Despite the higher richness found in GSB and GRC sites, few native perennial species with high coverage were recorded, due to dominating exotics and annuals. In contrast, grasslands used for pasture presented the typical vegetation features. For effective conservation efforts, we highlight the importance to keep grasslands on both soil types.

Keywords: crop rotation, Pampa, sandy soils

RESUMO - O uso da terra e a conservação da biodiversidade: um estudo de caso nos campos na Planície Costeira, Rio Grande do Sul. Os campos da região costeira estão entre os mais ameaçados do sul do Brasil. Para avaliar como áreas com diferentes históricos de uso variam quanto à composição e estrutura da vegetação, selecionamos quatro áreas: duas com Neossolos utilizadas para pecuária (áreas GR1 e GR2); e as demais com Planossolos em intervalo de pousio após cultivo de arroz (área GRC) e rotação soja/pastagem (área GSB). Em cada área, solo e vegetação campestre foram caracterizados. As áreas GR1, GSB, GRC e GR2 seguiram um padrão decrescente de riqueza. Apesar da alta riqueza em GSB e GRC, poucas espécies nativas perenes com alta cobertura foram registradas, havendo predomínio de exóticas e anuais. Já campos utilizados para pastejo mantiveram características típicas da região. Para assegurar a conservação desses campos é necessária a manutenção de áreas campestres sobre os dois tipos de solos.

Palavras-chave: neossolos, Pampa, rotação de culturas

\section{INTRODUCTION}

The supply of agricultural products and the providing of ecosystem services are essential for human existence and quality of life (Tilman et al. 2002). However, the intensification of agriculture has been the main cause of farmland biodiversity losses (Benton et al. 2003). The expansion of agriculture is responsible for soil degradation, changes in nutrient and carbon levels in the soil, as well as changes in its acidification and compaction (McLauchlan 2007), apart from effects on the distribution of plant and animal species (Benton et al. 2003). Changes in abiotic factors may leave an environmental legacy for the successional stages that follow, causing long-term changes in the composition and structure of vegetation even after land-use cessation In the case of grasslands, this fragmentation can even compromise its usability e.g. for livestock production, since it can contribute to the genetic erosion of forage grasses that are restricted to a given region (e.g. Medeiros et al. 2006).

The global trend of converting natural areas into agricultural areas can also be seen in the Pampa biome (sensu IBGE 2012) of southern Brazil. Roughly, 50\% of original vegetation has been converted for different land uses, and it is estimated that the conversion rate of natural and semi-natural grassland to anthropogenic grassland is of about 1,000 km²/year (Cordeiro \& Hasenack 2009). The grasslands on the Coastal Plain are especially affected by land use changes, converted mainly to rice crops. Today, we find in the region only $11 \%$ of natural grasslands, $8 \%$ degraded grasslands, $55 \%$ converted grasslands for different uses, and $26 \%$ remnansts of from other formations (Andrade et al. 2015).

The current land use in the Coastal Plain grassland s region is the result of socioeconomic, abiotic and biotic 
factors (Streck et al. 2008). In this region, large extensions of lowland soils have been used for rice production and extensive livestock grazing (Lopes et al. 2009). However, this scenario is currently changing by land use intensification especially by implementing a rotation system, such as soybeans/ryegrass production (IRGA 2013). Nonetheless, areas with low soil fertility and high erosion susceptibility remain grasslands. Given the high degree of landscape fragmentation and the modification of agricultural activity in the region, it is necessary to assess the extent to which the different types of land management can interfere with conservation of grasslands on the Coastal Plain. Specifically, it is important to understand to which extent native grasslands species can be re-established in fallow periods, which may last for one or more years in the case of rice crops but only months in the case of soybean rotation. In this current study, we analyze vegetation composition and structure in the Coastal Plain grasslands of Rio Grande do Sul, using for this, grasslands with different land-use histories.

\section{MATERIAL AND METHODS}

\section{Study area}

The study area is included in the geomorphological region of the southern Brazilian Coastal Plain of Rio Grande do Sul (Justus et al. 1986) in the municipality of São Lourenço do Sul ( $31^{\circ} 18^{\prime} \mathrm{S}, 51^{\circ} 58^{\prime} \mathrm{W}$, with mean an average elevation of $7 \mathrm{~m}$ a.s.1.). Monthly temperature means for the period from 1971 to 2000 was $17.8^{\circ} \mathrm{C}$ and monthly rainfall mean was $113.92 \mathrm{~mm}$ (Embrapa 2014). Our sampling was carried out in four sites with grassland vegetation belonging to a single farm that differ in terms of management:

\section{GSB (grassland/soybean)}

A 24 ha area originally covered by grassland, it is currently used for soybean crop during spring-summer and as pasture during winter seasons, with seeding of the exotic cool season species ryegrass (Lolium multiflorum L.). Two cycles of soybean had been completed before our sampling. The last soybean crop had been harvested six months before.

\section{GRC (grassland/rice crop)}

A 30 ha area originally covered by grassland, it contains a combination of rice crop during the spring and grazing cattle during the winter for more than fifty years. The last rice crop had been harvested six years ago, after this, the area was left to unassisted recovery and subjected to grazing by cattle livestock.

\section{GR1 (grassland 1) and GR2 (grassland 2)}

These two areas have eight and nine hectares, respectively. They are used for extensive livestock grazing, without record of other land uses.

\section{Soil sampling}

In order to characterize soil conditions, four composite samples were collected at a depth of up to $10 \mathrm{~cm}$ per area in October/2012. The following chemical properties were analyzed according to the methodology presented in Embrapa (1997): $\mathrm{pH}\left(\mathrm{H}_{2} \mathrm{O}\right)$, Potassium $\left(\mathrm{K}^{+}\right)$, Phosphorus (P), the exchangeable cations: Calcium $\left(\mathrm{Ca}^{+2}\right)$, Magnesium $\left(\mathrm{Mg}^{+2}\right)$ and Aluminum $\left(\mathrm{Al}^{+3}\right)$, soil organic matter (SOM) content and Cation Exchange Capacity (CEC). We also calculated the percentage of base saturation and aluminum saturation. One composite soil sample from each area was used for analyses of percentage of the different particle sizes (clay, fine sand, coarse sand and silt). Soils sampled were classified according to the Soil Survey Staff (2010) and Santos et al. (2006).

\section{Vegetation sampling}

During spring 2011, we analyzed the grassland plant community using 20 sampling units (SUs) of $0.25 \mathrm{~m}^{2}$ per area, arranged randomly (Matteucci \& Colma 1982). In each SU we estimated cover of each plant species, and the percentage of bare ground (BG), according to the scale proposed by Londo (1976). Quantitative and qualitative vegetation parameters as Absolute Frequency (AF), Relative Frequency (RF), Absolute Coverage (AC), Relative Coverage (RC) and Importance Value Index (IVI) were calculated for each species (Matteucci \& Colma 1982), and for BG separately. Information about life cycle and geographic distribution of species recorded was obtained from IBODA (2015).

When the identification of plant species was not possible in the field, samples were collected to confirm the identification by specialized literature. We followed Angiosperm Phylogeny Group IV (APG IV 2016).

\section{Data Analysis}

We visualized differences in vegetation patterns by Principal Coordinate Analysis, using chord distance as a dissimilarity measure between SUs. The significance of the axis of ordination was evaluated using the bootstrap technique (Pillar 2006). Only species that occurred with cover $>5 \%$, concerning all 80 sampling units, were used. Ryegrass was excluded from the matrix for this analysis, as its high cover in all SUs of GSB (grassland/soybean) limited the visualization of the pattern formed by the diagram for the other areas assessed. Species with high correlation to the axes were plotted in the diagram. The rescheduling of the environmental variable $\mathrm{BG}$ was performed by calculating the correlation between the BG along with scores of $\mathrm{SU}$ in the first two axes of ordination, then each value was multiplied by the average of the values of axis I and II, respectively.

Due to the high degradation of the studied environment and absence of comparable grassland fragments (regarding conservation status, land use history and study permission), we could not generate true replicates. However, this does not invalidate our study (Oksanen 2001), since all areas 
were homogeneous in topography (flat) and sufficiently large (between 10 and 50 hectares). Nonetheless, we did not conduct any formal hypothesis testing.

\section{RESULTS AND DISCUSSION}

Both areas with agricultural use, GSB (grassland/ soybean) and GRC (grassland/rice) were classified as Alfisols albaqualf (Soil Survey Staff 2010), and according to the Brazilian classification of soils (Santos et al. 2007) as "Planossolo háplico eutrófico". The area with no agricultural history GR1, was classified as Entisols quartzipsamments I and GR2 as Entisols quartzipsamments II (Soil Survey Staff 2010). According to the Brazilian classification of soils (Santos et al. 2007), the same areas were classified as "Neossolo quartzarênico órtico" and "Neossolo quartzarênico hidromórfico" respectively.

The Entisols areas evaluated (GR1 and GR2) are used for extensive livestock grazing, since they have low potential for crop production, mainly because of the low clay content (Tab. 1) that makes them sensitive to organic matter losses and erosion. The Alfisols areas (GSB and GRC), on the other hand, are traditionally used for rice production, because of the higher soil fertility and lowpermeability of the B-horizon. On sites with gentle slopes, soybean has recently started to be planted in the region. The high percentage of bases is probably due to residual fertility of previous agricultural activities. On the other hand, SOM and $\mathrm{Al}+\mathrm{H}$ were highest in areas without agricultural activities (see Tab. 2).

Considering all four areas, we recorded 126 plant species from 37 families. The families with most species were Poaceae (24 spp.), Asteraceae (23 spp.) and Cyperaceae (12 spp.). Among the areas evaluated, GR1 area had the highest number of species (73 spp.), followed by GSB and GRC (both with $61 \mathrm{spp}$.). The lowest number of species was recorded for area GR2 (56 spp.). The species with the highest IVI values differed among the areas. In GSB, the highest IVI values were recorded for Lolium multiflorum Lam., Oxalis debilis Kunth, Oxalis bipartita A. St.-Hil., and Axonopus affinis Chase. In GRC, the highest IVI values were recorded for A. affinis, Lobelia hederacea Cham., Centella asiatica (L.) Urb., and Eleocharis viridans Kük. ex Osten. In GR1, the highest IVI values were recorded for Paspalum pumilum Ness, A. affinis, L. hederacea, and Setaria vaginata Spreng. Important species in GR2 were P. pumilum, Sphagnum sp., A. affinis and Panicum aquaticum Poir. (Tab. 3).

The dominant species in the GSB area was $L$. multiflorum, used as forage grass during the winter. Native annual and exotic species were the main components in the plant community, while species typical for grasslands were absent or had low IVI values (Tab. 3), reflecting the prevalence of ruderal species in these disturbed areas (Grime 2002). The ryegrass dominance probably hampers the establishment of typical grasslands species (Bellemare et al. 2002) by shading, as well as low soil nutrient and water shortages (Grime 2002).

The other Alfisols area GRC had high cover of $A$. affinis, a grass of wide distribution in the Rio Grande do Sul grasslands (Boldrini \& Longhi-Wagner 2011). Dominance of the species in the area that has recovered to grassland after rice production probably is a consequence of its strong vegetative propagation by stolons (Coughneour 1985). One can expect dominance by generalist species, such as $A$. affinis, in fragmented habitats or in sites with disturbance history (Harrison \& Emilio 1999). The results for the GRC are similar to a study conducted by Garcia (2005) in a similar Alfisols area with 4-year fallow history (Tab. 4).

In areas GR1 and GR2, the higher IVI of typical grassland species was associated with the lower IVI of annual and exotic species, related to disturbance and corresponding life-history characteristics of the plants.

Table 1. Soil texture for the four areas with different land-use history, São Lourenço do Sul, RS. GSB = grassland of soybean/ryegrass, GRC = grassland rice/livestock. GR1 and GR2 = grassland used for extensive livestock breeding.

\begin{tabular}{lcccc}
\hline Soil textural classes & GSB & GRC & GR1 & GR2 \\
\hline Clay (\%) & 12.25 & 13 & 7.75 & 7.25 \\
Silt (\%) & 39 & 44 & 14 & 5 \\
Fine sand (\%) & 19 & 19 & 65 & 83 \\
Coarse sand (\%) & 29 & 24 & 12 & 5 \\
\hline
\end{tabular}

Table 2. Values of soil chemical variables of the four areas with different land-use history, São Lourenço do Sul, RS. GSB = grassland of soybean/ryegrass, GRC = grassland rice/livestock. GR1 and GR2 = grassland used for extensive livestock breeding.

\begin{tabular}{|c|c|c|c|c|}
\hline Average values of the chemical analysis & GSB & GRC & GR1 & GR2 \\
\hline $\mathrm{pH}\left(\mathrm{H}_{2} \mathrm{O}\right)$ & 5.22 & 5.32 & 4.62 & 4.57 \\
\hline Soil organic matter (SOM) & 2.27 & 2.22 & 6.67 & 5.75 \\
\hline Bases (\% SAT da CTC) & 63.25 & 57.75 & 9.75 & 9.75 \\
\hline $\mathrm{P}\left(\mathrm{mg} / \mathrm{dm}^{3}\right)$ & 11.72 & 6.32 & 8 & 6.15 \\
\hline $\mathrm{K}\left(\mathrm{mg} / \mathrm{dm}^{3}\right)$ & 36.75 & 63 & 145.5 & 40 \\
\hline $\mathrm{Ca}^{+2}\left(\mathrm{cmolc} / \mathrm{dm}^{3}\right)$ & 3.3 & 2.35 & 0.8 & 0.9 \\
\hline $\mathrm{Mg}^{+2}\left(\mathrm{cmolc} / \mathrm{dm}^{3}\right)$ & 1.82 & 1.37 & 0.35 & 0.37 \\
\hline $\mathrm{Al}+\mathrm{H}\left(\mathrm{cmolc} / \mathrm{dm}^{3}\right)$ & 2.97 & 2.87 & 15.15 & 10.92 \\
\hline $\mathrm{CTC}\left(\mathrm{cmolc} / \mathrm{dm}^{3}\right)$ & 8.25 & 6.77 & 16.7 & 12.3 \\
\hline $\mathrm{Al}\left(\mathrm{cmolc} / \mathrm{dm}^{3}\right)(\%$ SAT da CTC $)$ & 3.95 & 5.12 & 55.7 & 63.67 \\
\hline
\end{tabular}


Table 3. Quantitative parameters of vegetation survey recorded through 80 sampling units in four grassland areas with different land-use history. São Lourenço do Sul, RS. Threat rating from the list of endangered flora of Rio Grande do Sul (SEMA 2003). RF $=$ relative frequency, RC = relative coverage, IVI = importance value index, $\mathrm{EN}=$ Endangered, $\mathrm{VU}=$ Vulnerable, $*$ annual, ** exotic species, *** annual and exotic species.

\begin{tabular}{|c|c|c|c|c|c|c|c|c|c|c|c|c|}
\hline \multirow{2}{*}{ Soil classification } & \multicolumn{3}{|c|}{$\begin{array}{l}\text { Grassland of soybean/ } \\
\text { ryegrass }\end{array}$} & \multicolumn{3}{|c|}{$\begin{array}{c}\text { Grassland of rice/ } \\
\text { livestock }\end{array}$} & \multicolumn{3}{|c|}{$\begin{array}{l}\text { Grassland used for } \\
\text { livestock } 1\end{array}$} & \multicolumn{3}{|c|}{$\begin{array}{c}\text { Grassland used for } \\
\text { livestock } 2\end{array}$} \\
\hline & RF & $\mathrm{RC}$ & IVI & RF & $\mathrm{RC}$ & IVI & RF & $\mathrm{RC}$ & IVI & RF & $\mathrm{RC}$ & IVI \\
\hline Bare soil & 5.19 & 26.42 & 16.17 & 5.30 & 33.17 & 19.24 & 7.22 & 12.60 & 9.91 & 8.43 & 5.94 & 7.18 \\
\hline \multicolumn{13}{|l|}{ Families/species } \\
\hline \multicolumn{13}{|l|}{ Anthocerotaceae } \\
\hline Anthoceros sp. & 1.89 & 0.37 & 1.13 & - & - & - & - & - & - & - & - & - \\
\hline \multicolumn{13}{|l|}{ Alismataceae } \\
\hline Echinodorus longiscapus Arech. & - & - & - & 0.28 & 0.07 & 0.17 & - & - & - & - & - & - \\
\hline \multicolumn{13}{|l|}{ Amaranthaceae } \\
\hline Alternanthera philoxeroides (Mart.) Griseb. & - & - & - & - & - & - & 0.39 & 0.06 & 0.23 & - & - & - \\
\hline Pfaffia tuberosa Hicken & - & - & - & 2.24 & 0.81 & 1.53 & 0.39 & 0.06 & 0.23 & - & - & - \\
\hline \multicolumn{13}{|l|}{ Apiaceae } \\
\hline Centella asiatica (L.) Urb. & 5.97 & 2.18 & 4.08 & 4.76 & 6.08 & 5.42 & 4.72 & 3.29 & 4.01 & 5.07 & 1.51 & 3.29 \\
\hline Eryngium divaricatum Hook. \& Arn. (VU) & - & - & - & 1.4 & 0.88 & 1.14 & - & - & - & - & - & - \\
\hline E. elegans Cham. \& Schltdl. & - & - & - & - & - & - & 0.79 & 0.6 & 0.69 & - & - & - \\
\hline E. nudicaule Lam. & - & - & - & - & - & - & 0.39 & 0.06 & 0.23 & - & - & - \\
\hline Lilaeopsis tenuis A.W. Hill & 0.31 & 0.06 & 0.19 & - & - & - & 0.39 & 0.06 & 0.23 & - & - & - \\
\hline \multicolumn{13}{|l|}{ Araliaceae } \\
\hline Hydrocotyle bonariensis Lam. & 1.26 & 0.25 & 0.75 & - & - & - & 4.72 & 0.72 & 2.72 & 1.38 & 0.53 & 0.96 \\
\hline H. exigua Malme & - & - & - & 0.28 & 0.07 & 0.17 & 0.79 & 0.12 & 0.45 & 0.46 & 0.05 & 0.25 \\
\hline H. ranunculoides L. f. & 1.26 & 0.25 & 0.75 & - & - & - & 0.79 & 0.12 & 0.45 & - & - & - \\
\hline \multicolumn{13}{|l|}{ Asteraceae } \\
\hline Aspilia montevidensis (Spreng.) Kuntze & - & - & - & - & - & - & - & - & - & 0.46 & 0.05 & 0.25 \\
\hline $\begin{array}{l}\text { Baccharis genistelloides subsp. crispa } \\
\text { (Spreng.) Joch. Müll. }\end{array}$ & - & - & - & - & - & - & 0.79 & 0.36 & 0.57 & 0.46 & 0.24 & 0.35 \\
\hline Chaptalia runcinata Kunth & - & - & - & 0.28 & 0.07 & 0.17 & 5.12 & 2.04 & 3.58 & 2.76 & 0.88 & 1.82 \\
\hline Coleostephus myconis (L.) Cass. & 0.31 & 0.06 & 0.19 & - & - & - & 1.57 & 0.72 & 1.15 & - & - & - \\
\hline Conyza bonariensis (L.) Cronquist* & 1.26 & 0.25 & 0.75 & - & - & - & 0.79 & 0.12 & 0.45 & - & - & - \\
\hline Elephantopus mollis Kunth & - & - & - & - & - & - & 1.18 & 0.42 & 0.8 & 0.46 & 0.24 & 0.35 \\
\hline Facelis retusa (Lam.) Sch. Bip.* & 0.31 & 0.06 & 0.19 & - & - & - & 3.15 & 0.48 & 1.81 & 0.92 & 0.1 & 0.51 \\
\hline Gamochaeta americana (Mill.) Wedd. & 3.14 & 0.62 & 1.88 & 0.84 & 0.2 & 0.52 & 1.97 & 1.02 & 1.49 & 0.46 & 0.24 & 0.35 \\
\hline G. coarctata (Willd.) Kerguélen & 2.52 & 2.62 & 2.57 & 4.2 & 2.16 & 3.18 & 1.18 & 0.42 & 0.8 & 0.46 & 0.05 & 0.25 \\
\hline G. filaginea (DC.) Cabrera & 0.63 & 0.12 & 0.38 & - & - & - & - & - & - & - & - & - \\
\hline $\begin{array}{l}\text { Hypochaeris albiflora (Kuntze) Azevêdo- } \\
\text { Gonç. \& Matzenb. }\end{array}$ & 0.31 & 0.06 & 0.19 & - & - & - & - & - & - & - & - & - \\
\hline H. chillensis (Kunth) Britton & - & - & - & 1.96 & 0.47 & 1.22 & - & - & - & 0.46 & 0.05 & 0.25 \\
\hline H. glabra $\mathrm{L}$ & 0.63 & 0.12 & 0.38 & 0.28 & 0.07 & 0.17 & 0.79 & 0.66 & 0.72 & - & - & - \\
\hline H. megapotamica Cabrera & 0.63 & 0.12 & 0.38 & 0.28 & 0.07 & 0.17 & 0.39 & 0.06 & 0.23 & 1.38 & 0.53 & 0.96 \\
\hline Micropsis spathulata (Pers.) Cabrera* & 2.52 & 1.81 & 2.16 & 4.48 & 1.08 & 2.78 & - & - & - & 1.38 & 0.34 & 0.86 \\
\hline Noticastrum calvatum (Baker) Cuatrec. & - & - & - & - & - & - & 0.39 & 0.06 & 0.23 & - & - & - \\
\hline Pluchea sagittalis (Lam.) Cabrera* & 0.63 & 0.12 & 0.38 & 3.08 & 0.74 & 1.91 & - & - & - & - & - & - \\
\hline Pterocaulon alopecuroides (Lam.) DC & - & - & - & - & - & - & 1.18 & 0.96 & 1.07 & - & - & - \\
\hline Senecio selloi (Spreng.) DC. & - & - & - & - & - & - & 1.97 & 1.08 & 1.52 & - & - & - \\
\hline Soliva macrocephala Cabrera* & 1.89 & 0.37 & 1.13 & 1.68 & 0.95 & 1.31 & - & - & - & - & - & - \\
\hline S. sessilis Ruiz \& Pav.* & 3.14 & 1.75 & 2.45 & 2.52 & 0.61 & 1.56 & 2.76 & 0.9 & 1.83 & 1.38 & 0.58 & 0.98 \\
\hline $\begin{array}{l}\text { Symphyotrichum squamatum (Spreng.) G.L. } \\
\text { Nesom* }\end{array}$ & 2.52 & 1.06 & 1.79 & 3.36 & 1.08 & 2.22 & - & - & - & - & - & - \\
\hline \multicolumn{13}{|l|}{ Calyceraceae } \\
\hline Acicarpha procumbens Less.* & - & - & - & - & - & - & 0.79 & 0.9 & 0.84 & - & - & - \\
\hline \multicolumn{13}{|l|}{ Campanulaceae } \\
\hline Lobelia hederacea Cham. & 2.2 & 1.5 & 1.85 & 3.92 & 7.36 & 5.64 & 1.97 & 4.85 & 3.41 & 0.46 & 0.05 & 0.25 \\
\hline \multicolumn{13}{|l|}{ Caryophyllaceae } \\
\hline
\end{tabular}


Table 3. Cont.

\begin{tabular}{|c|c|c|c|c|c|c|c|c|c|c|c|c|}
\hline \multirow{2}{*}{ Soil classification } & \multicolumn{3}{|c|}{$\begin{array}{l}\text { Grassland of soybean/ } \\
\text { ryegrass }\end{array}$} & \multicolumn{3}{|c|}{$\begin{array}{l}\text { Grassland of rice/ } \\
\text { livestock }\end{array}$} & \multicolumn{3}{|c|}{$\begin{array}{l}\text { Grassland used for } \\
\text { livestock } 1\end{array}$} & \multicolumn{3}{|c|}{$\begin{array}{l}\text { Grassland used for } \\
\text { livestock } 2\end{array}$} \\
\hline & $\mathrm{RF}$ & $\mathrm{RC}$ & IVI & $\mathrm{RF}$ & $\mathrm{RC}$ & IVI & $\mathrm{RF}$ & $\mathrm{RC}$ & IVI & $\mathrm{RF}$ & $\mathrm{RC}$ & IVI \\
\hline Drymaria cordata (L.) Willd. ex Roem. \& Schult* & - & - & - & - & - & - & 1.18 & 0.42 & 0.8 & - & - & - \\
\hline Sagina chilensis Naud. ex Gay*** & 1.26 & 0.25 & 0.75 & 3.92 & 0.95 & 2.43 & - & - & - & 0.92 & 0.1 & 0.51 \\
\hline Spergula arvensis $\mathrm{L} . * * *$ & - & - & - & 0.28 & 0.07 & 0.17 & 0.39 & 0.06 & 0.23 & 0.46 & 0.05 & 0.25 \\
\hline Stellaria media (L.) Vill.*** & 0.63 & 0.12 & 0.38 & - & - & - & - & - & - & - & - & - \\
\hline \multicolumn{13}{|l|}{ Convolvulaceae } \\
\hline Dichondra sericea Sw. & - & - & - & - & - & - & 1.18 & 0.96 & 1.07 & - & - & - \\
\hline \multicolumn{13}{|l|}{ Cyperaceae } \\
\hline Bulbostylis capillaris (L.) C.B. Clarke & - & - & - & 0.56 & 0.14 & 0.35 & - & - & - & 0.92 & 0.29 & 0.61 \\
\hline Eleocharis bonariensis Nees & 1.26 & 2 & 1.63 & 1.4 & 1.89 & 1.65 & - & - & - & 0.46 & 0.24 & 0.35 \\
\hline E. sellowiana Kunth & 0.31 & 0.06 & 0.19 & 0.56 & 1.42 & 0.99 & 0.39 & 0.6 & 0.5 & - & - & - \\
\hline E. viridans Kük. ex Osten & 1.57 & 2.12 & 1.85 & 4.2 & 4.8 & 4.5 & 0.39 & 0.3 & 0.35 & 0.46 & 0.49 & 0.47 \\
\hline Kyllinga brevifolia Rottb. & 0.31 & 0.06 & 0.19 & 0.28 & 0.07 & 0.17 & 0.79 & 0.6 & 0.69 & 0.46 & 0.24 & 0.35 \\
\hline K. odorata Vahl & 1.26 & 0.5 & 0.88 & 0.28 & 0.07 & 0.17 & 1.97 & 1.92 & 1.94 & - & - & - \\
\hline K. vaginata Lam. & - & - & - & - & - & - & - & - & - & 0.46 & 0.24 & 0.35 \\
\hline Pycreus polystachyos (Rottb.) P. Beauv. & 0.94 & 0.19 & 0.57 & 0.56 & 0.41 & 0.48 & 1.97 & 1.26 & 1.61 & 4.61 & 2.14 & 3.37 \\
\hline Rhynchospora barrosiana Guagl. & - & - & - & - & - & - & - & - & - & 0.92 & 0.73 & 0.83 \\
\hline R. holoschoenoides (Rich.) Herter & - & - & - & - & - & - & 1.97 & 0.84 & 1.4 & 3.69 & 0.58 & 2.14 \\
\hline R. tenuis Willd. ex Link & - & - & - & 0.84 & 0.2 & 0.52 & 4.72 & 2.76 & 3.74 & 5.99 & 0.83 & 3.41 \\
\hline Scleria distans Poir. & - & - & - & - & - & - & - & - & - & 2.3 & 0.44 & 1.37 \\
\hline \multicolumn{13}{|l|}{ Droseraceae } \\
\hline Drosera brevifolia Pursh & - & - & - & - & - & - & 0.39 & 0.3 & 0.35 & 3.23 & 0.73 & 1.98 \\
\hline \multicolumn{13}{|l|}{ Fabaceae } \\
\hline Aeschynomene denticulta Rudd. & - & - & - & 0.56 & 0.14 & 0.35 & - & - & - & - & - & - \\
\hline Desmodium incanum DC. & 0.31 & 0.06 & 0.19 & - & - & - & - & - & - & - & - & - \\
\hline Macroptilium prostratum (Benth.) Urb. & 0.31 & 0.31 & 0.31 & - & - & - & - & - & - & - & - & - \\
\hline Medicago lupulina $\mathrm{L} . * * *$ & 0.63 & 0.62 & 0.63 & - & - & - & - & - & - & - & - & - \\
\hline Trifolium repens L.*** & 4.72 & 3.05 & 3.89 & - & - & - & - & - & - & - & - & - \\
\hline \multicolumn{13}{|l|}{ Haloragaceae } \\
\hline Laurembergia tetrandra (Schott) Kanitz (EN) & - & - & - & 0.28 & 0.07 & 0.17 & - & - & - & 1.38 & 0.34 & 0.86 \\
\hline \multicolumn{13}{|l|}{ Hypoxidaceae } \\
\hline $\begin{array}{l}\text { Hypoxis decumbens } \mathrm{L} . \\
\text { Iridaceae }\end{array}$ & 0.63 & 0.12 & 0.38 & 0.28 & 0.07 & 0.17 & 1.57 & 0.72 & 1.15 & - & - & - \\
\hline $\begin{array}{l}\text { Sisyrinchium micranthum Cav. } \\
\text { Juncaceae }\end{array}$ & 2.52 & 0.5 & 1.51 & 3.36 & 1.08 & 2.22 & 1.97 & 0.3 & 1.13 & 1.84 & 0.19 & 1.02 \\
\hline Juncus bufonius L. & 3.46 & 1.18 & 2.32 & 3.08 & 1.89 & 2.49 & - & - & - & - & - & - \\
\hline J. microcephalus Kunth & 3.77 & 1.56 & 2.67 & 4.48 & 1.89 & 3.19 & 0.39 & 0.06 & 0.23 & 0.46 & 0.05 & 0.25 \\
\hline J. tenuis Willd. & - & - & - & 0.56 & 0.41 & 0.48 & 0.39 & 0.06 & 0.23 & - & - & - \\
\hline \multicolumn{13}{|l|}{ Juncaginaceae } \\
\hline Lilaea scilloides (Poir.) Hauman (EN) & - & - & - & 1.12 & 0.27 & 0.7 & - & - & - & - & - & - \\
\hline \multicolumn{13}{|l|}{ Lamiaceae } \\
\hline \multicolumn{13}{|l|}{ Lycopodiaceae } \\
\hline \multicolumn{13}{|l|}{ Lythraceae } \\
\hline Lythrum hyssopifolia L. & - & - & - & 0.28 & 2.03 & 1.15 & - & - & - & 1.84 & 0.39 & 1.12 \\
\hline Malvaceae & & & & & & & & & & & & \\
\hline Sida rhombifolia $\mathrm{L}$. & - & - & - & 0.28 & 0.07 & 0.17 & - & - & - & - & - & - \\
\hline Melastomataceae & & & & & & & & & & & & \\
\hline Tibouchina asperior (Cham.) Cogn. (EN) & - & - & - & - & - & - & 1.18 & 0.18 & 0.68 & 0.46 & 0.05 & 0.25 \\
\hline Menyanthaceae & & & & & & & & & & & & \\
\hline Nymphoides indica (L.) Kuntze & - & - & - & 0.28 & 0.68 & 0.48 & - & - & - & 0.92 & 0.29 & 0.61 \\
\hline Onagraceae & & & & & & & & & & & & \\
\hline $\begin{array}{l}\text { Ludwigia hexapetala (Hook. \& Arn.) Zardini, } \\
\text { H. Gu \& P.H. Raven }\end{array}$ & 0.31 & 0.06 & 0.19 & - & - & - & - & - & - & - & - & - \\
\hline Ophioglossaceae & & & & & & & & & & & & \\
\hline
\end{tabular}


Table 3. Cont.

\begin{tabular}{|c|c|c|c|c|c|c|c|c|c|c|c|c|}
\hline \multirow{2}{*}{ Soil classification } & \multicolumn{3}{|c|}{$\begin{array}{l}\text { Grassland of soybean/ } \\
\text { ryegrass }\end{array}$} & \multicolumn{3}{|c|}{$\begin{array}{c}\text { Grassland of rice/ } \\
\text { livestock }\end{array}$} & \multicolumn{3}{|c|}{$\begin{array}{l}\text { Grassland used for } \\
\text { livestock } 1\end{array}$} & \multicolumn{3}{|c|}{$\begin{array}{l}\text { Grassland used for } \\
\text { livestock } 2\end{array}$} \\
\hline & $\mathrm{RF}$ & $\mathrm{RC}$ & IVI & $\mathrm{RF}$ & $\mathrm{RC}$ & IVI & $\mathrm{RF}$ & $\mathrm{RC}$ & IVI & $\mathrm{RF}$ & $\mathrm{RC}$ & IVI \\
\hline Ophioglossum crotalophoroides Walter & 0.63 & 0.12 & 0.38 & - & - & - & - & - & - & - & - & - \\
\hline \multicolumn{13}{|l|}{ Orobanchaceae } \\
\hline Buchnera longiflora Arn. & - & - & - & 0.56 & 0.14 & 0.35 & - & - & - & - & - & - \\
\hline \multicolumn{13}{|l|}{ Oxalidaceae } \\
\hline Oxalis bipartita A. St.-Hil. & 4.4 & 4.68 & 4.54 & 2.24 & 1.69 & 1.96 & 0.39 & 0.6 & 0.5 & - & - & - \\
\hline O. debilis Kunth & 5.35 & 6.23 & 5.79 & 2.8 & 2.36 & 2.58 & - & - & - & - & - & - \\
\hline \multicolumn{13}{|l|}{ Plantaginaceae } \\
\hline Bacopa monnieri (L.) Wettst.* & 1.26 & 0.25 & 0.75 & 0.84 & 0.2 & 0.52 & - & - & - & 0.92 & 0.53 & 0.73 \\
\hline Callitriche deflexa A. Braun ex Hegelm.* & 1.89 & 0.37 & 1.13 & 1.12 & 0.27 & 0.7 & - & - & - & - & - & - \\
\hline Gratiola peruviana L.* & 0.63 & 0.12 & 0.38 & 0.84 & 0.2 & 0.52 & 0.39 & 0.06 & 0.23 & - & - & - \\
\hline Linaria texana Scheele* & - & - & - & - & - & - & - & - & - & 0.46 & 0.05 & 0.25 \\
\hline Plantago australis Lam. & 0.31 & 0.06 & 0.19 & - & - & - & 0.79 & 0.36 & 0.57 & 0.46 & 0.49 & 0.47 \\
\hline P. myosuros Lam. & 1.57 & 0.31 & 0.94 & 0.28 & 0.07 & 0.17 & 0.39 & 0.3 & 0.35 & - & - & - \\
\hline \multicolumn{13}{|l|}{ Poaceae } \\
\hline Andropogon lateralis Nees & - & - & - & - & - & - & 0.39 & 0.06 & 0.23 & - & - & - \\
\hline A. selloanus (Hack.) Hack. & 0.31 & 0.06 & 0.19 & 1.12 & 0.54 & 0.83 & 1.57 & 1.56 & 1.57 & 3.69 & 4.42 & 4.06 \\
\hline Axonopus affinis Chase & 4.72 & 3.99 & 4.35 & 5.04 & 33.98 & 19.51 & 6.3 & 17.55 & 11.93 & 6.91 & 10.55 & 8.73 \\
\hline Briza minor L.*** & 0.31 & 0.06 & 0.19 & - & - & - & - & - & - & - & - & - \\
\hline Cynodon dactylon (L.) Pers.** & 0.63 & 0.12 & 0.38 & 1.96 & 2.3 & 2.13 & - & - & - & - & - & - \\
\hline $\begin{array}{l}\text { Dichanthelium sabulorum (Lam.) Gould \& } \\
\text { C.A. Clark }\end{array}$ & - & - & - & - & - & - & 0.79 & 0.6 & 0.69 & - & - & - \\
\hline Eragrostis cataclasta Nicora & - & - & - & - & - & - & - & - & - & 2.3 & 1.07 & 1.69 \\
\hline E. neesii Trin. & - & - & - & - & - & - & 0.39 & 0.06 & 0.23 & - & - & - \\
\hline Ischaemum minus J. Presl & - & - & - & 1.96 & 2.16 & 2.06 & 0.79 & 0.9 & 0.84 & 3.23 & 5.4 & 4.31 \\
\hline Lolium multiflorum Lam.*** & 6.29 & 54.24 & 30.26 & - & - & - & - & - & - & - & - & - \\
\hline Luziola peruviana Juss. ex J.F. Gmel. & - & - & - & 1.96 & 3.11 & 2.53 & - & - & - & 0.46 & 1.46 & 0.96 \\
\hline Panicum aquaticum Poir. & - & - & - & - & - & - & 1.97 & 1.98 & 1.97 & 5.07 & 3.74 & 4.41 \\
\hline P. dichotomiflorum Michx. & - & - & - & 1.96 & 0.74 & 1.35 & 0.79 & 0.9 & 0.84 & - & - & - \\
\hline Paspalum dilatatum Poir. & - & - & - & - & - & - & 0.39 & 0.06 & 0.23 & - & - & - \\
\hline$P$. notatum Flüggé & - & - & - & 0.56 & 0.74 & 0.65 & 0.39 & 0.3 & 0.35 & - & - & - \\
\hline P. plicatulum Michx. & - & - & - & - & - & - & 0.39 & 0.6 & 0.5 & - & - & - \\
\hline P. pumilum Nees & 0.63 & 0.12 & 0.38 & 1.96 & 4.39 & 3.18 & 4.72 & 29.65 & 17.19 & 7.37 & 42.29 & 24.83 \\
\hline P. vaginatum $\mathrm{Sw}$. & - & - & - & - & - & - & 2.36 & 4.91 & 3.64 & 0.46 & 0.97 & 0.72 \\
\hline Poa annua L.*** & 1.57 & 0.31 & 0.94 & 1.12 & 0.81 & 0.97 & - & - & - & - & - & - \\
\hline $\begin{array}{l}\text { Schizachyrium microstachyum (Desv. ex } \\
\text { Ham.) Roseng., B.R. Arrill. \& Izag. }\end{array}$ & - & - & - & - & - & - & - & - & - & 0.92 & 1.22 & 1.07 \\
\hline Setaria parviflora (Poir.) Kerguélen & - & - & - & 0.28 & 0.07 & 0.17 & - & - & - & - & - & - \\
\hline S. vaginata Spreng. & - & - & - & - & - & - & 3.94 & 4.67 & 4.3 & - & - & - \\
\hline Sporobolus indicus (L.) Br. & - & - & - & 0.28 & 0.07 & 0.17 & - & - & - & 0.92 & 0.1 & 0.51 \\
\hline Steinchisma hians (Elliott) Nash & 0.63 & 0.12 & 0.38 & 0.28 & 0.07 & 0.17 & 1.18 & 0.42 & 0.8 & 2.76 & 0.29 & 1.53 \\
\hline \multicolumn{13}{|l|}{ Polygonaceae } \\
\hline Polygonum punctatum Elliott & 0.31 & 0.06 & 0.19 & - & - & - & - & - & - & - & - & - \\
\hline \multicolumn{13}{|l|}{ Primulaceae } \\
\hline Centunculus minimus L.* & 4.4 & 0.87 & 2.64 & 3.92 & 1.55 & 2.74 & 1.18 & 0.42 & 0.8 & 0.92 & 0.1 & 0.51 \\
\hline \multicolumn{13}{|l|}{ Ranunculaceae } \\
\hline Ranunculus bonariensis Poir.* & 1.89 & 0.37 & 1.13 & 4.48 & 2.63 & 3.56 & - & - & - & - & - & - \\
\hline \multicolumn{13}{|l|}{ Rubiaceae } \\
\hline Galium humile Cham. \& Schltdl. & - & - & - & - & - & - & 2.36 & 0.36 & 1.36 & 0.46 & 0.05 & 0.25 \\
\hline $\begin{array}{l}\text { Richardia humistrata (Cham. \& Schltdl.) } \\
\text { Steud. }\end{array}$ & - & - & - & - & - & - & 0.39 & 0.06 & 0.23 & 0.46 & 0.24 & 0.35 \\
\hline R. stellaris (Cham. \& Schltdl.) Steud. & - & - & - & - & - & - & 0.39 & 0.06 & 0.23 & - & - & - \\
\hline Spermacoce verticillata $\mathrm{L}$. & - & - & - & - & - & - & 0.39 & 0.3 & 0.35 & - & - & - \\
\hline \multicolumn{13}{|l|}{ Solanaceae } \\
\hline Schwenckia curviflora Benth. & - & - & - & - & - & - & - & - & - & 1.38 & 0.15 & 0.76 \\
\hline Solanum americanum Mill. & - & - & - & - & - & - & 1.57 & 1.02 & 1.3 & 1.84 & 0.19 & 1.02 \\
\hline
\end{tabular}


Table 3. Cont.

\begin{tabular}{|c|c|c|c|c|c|c|c|c|c|c|c|c|}
\hline \multirow[t]{2}{*}{ Soil classification } & \multicolumn{3}{|c|}{$\begin{array}{c}\text { Grassland of soybean/ } \\
\text { ryegrass }\end{array}$} & \multicolumn{3}{|c|}{$\begin{array}{l}\text { Grassland of rice/ } \\
\text { livestock }\end{array}$} & \multicolumn{3}{|c|}{$\begin{array}{l}\text { Grassland used for } \\
\text { livestock } 1\end{array}$} & \multicolumn{3}{|c|}{$\begin{array}{l}\text { Grassland used for } \\
\text { livestock } 2\end{array}$} \\
\hline & $\mathrm{RF}$ & $\mathrm{RC}$ & IVI & RF & $\mathrm{RC}$ & IVI & $\mathrm{RF}$ & $\mathrm{RC}$ & IVI & $\mathrm{RF}$ & $\mathrm{RC}$ & IVI \\
\hline S. sisymbriifolium Lam. & 0.31 & 0.06 & 0.19 & - & - & - & 0.79 & 0.12 & 0.45 & - & - & - \\
\hline \multicolumn{13}{|l|}{ Sphagnaceae } \\
\hline Sphagnum sp. & 0.63 & 0.12 & 0.38 & 0.84 & 0.22 & 0.53 & 1.18 & 0.2 & 0.69 & 7.83 & 12.49 & 10.16 \\
\hline \multicolumn{13}{|l|}{ Verbenaceae } \\
\hline Glandularia selloi (Spreng.) Tronc. & 1.89 & 0.37 & 1.13 & 0.28 & 0.34 & 0.31 & - & - & - & - & - & - \\
\hline Verbena montevidensis Spreng. & - & - & - & - & - & - & 0.39 & 0.06 & 0.23 & - & - & - \\
\hline \multicolumn{13}{|l|}{ Lichenized fungii } \\
\hline Cladonia sp. & - & - & - & - & - & - & - & - & - & 0.46 & 0.05 & 0.25 \\
\hline
\end{tabular}

Table 4. Species and bare soil with higher Importance Value (IVI) in different areas of the Coastal Plain grasslands in Rio Grande do Sul. Capivari do Sul (Garcia 2005), Santo Antônio da Patrulha (Ferreira \& Setubal 2009), Osório (Boldrini et al. 2008), and this study GR1 and GR2. = grassland used for extensive livestock breeding. $*=$ highest values.

\begin{tabular}{|c|c|c|c|c|c|}
\hline Survey's Areas & Capivari do Sul & $\begin{array}{l}\text { Santo Antonio } \\
\text { da Patrulha }\end{array}$ & Osório & $\begin{array}{l}\text { São Lourenço do Sul } \\
\text { (GR1) }\end{array}$ & $\begin{array}{l}\text { São Lourenço do Sul } \\
\text { (GR2) }\end{array}$ \\
\hline Andropogon lateralis & - & - & 5.89 & 0.23 & - \\
\hline Axonopus affinis & 5.01 & $7.01 *$ & - & $11.93 *$ & $8.73 *$ \\
\hline A. parodii & $12.75^{*}$ & - & $10.65^{*}$ & - & - \\
\hline Centella asiatica & $20.5^{*}$ & 4.26 & 2.22 & 4.01 & 3.29 \\
\hline Desmodium incanum & - & $4.71^{*}$ & 1.08 & - & - \\
\hline Eleocharis viridans & 4.67 & 0.55 & 0.60 & 0.35 & 0.47 \\
\hline Ischaemum minus & $17.41^{*}$ & $4.71 *$ & $7.46^{*}$ & 0.84 & 4.31 \\
\hline Panicum aquaticum & - & - & 1.68 & 1.97 & 4.41 \\
\hline Paspalum lepton & 2.75 & 1.74 & $5.83 *$ & - & - \\
\hline P. notatum & $45.5^{*}$ & $10.25^{*}$ & - & 0.35 & - \\
\hline P. pumilum & 6.19 & 3.39 & $6.99 *$ & $17.19^{*}$ & $24.83^{*}$ \\
\hline Setaria vaginata & - & - & - & $4.3^{*}$ & - \\
\hline Sphagnum sp. & - & - & 2.95 & 0.69 & $10.16^{*}$ \\
\hline Bare soil & 5 & - & 1.14 & $9.91 *$ & $7.18^{*}$ \\
\hline
\end{tabular}

Some species, such as Andropogon selloanus (Hack.) Hack., Ischaemum minus J. Presl, P. pumilum, and Rhynchospora tenuis Willd. ex Link that had IVI values in areas on Entisols, are indicative of well-managed grasslands, linking extensive livestock grazing with biodiversity conservation (see Boldrini 2009).

Vegetation of the two areas on Entisols that are used for extensive livestock production (GR1 and GR2) are in agreement with other studies of the coastal plain grasslands in southern Brazil (Boldrini \& Longhi-Wagner 2011, Garcia 2005, Boldrini et al. 2008, Ferreira \& Setubal 2009) in terms of grasslands physiognomy and structure, species richness and the highest IVI species values (Tab. 4). The two areas differ in their structure probably due to the higher water content in the soil in GR2, pointed by the high Sphagnum sp. covering (Li et al. 2004). This extreme condition is also reflected in the lower species richness of this area (Tab. 3).

Annual and exotic species were probably favored due to adaptive strategies for disturbed areas: low height, annual life cycle and high production annual seed (Grime 2002). The Importance Value Index (IVI) reflects this tendency. In GSB, annual species represent the highest percentage $52.49 \%$ of IVI and exotic species $37.99 \%$. In GRC, annual species represent $21.39 \%$ and exotic species $5.7 \%$. In the
GR1, annual species totalizing 7.45\% and only three exotic species represent $0.46 \%$, and in the GR2, annual species $4.6 \%$ and exotic species $0.76 \%$ (Table 2). Regarding the similarity between the areas evaluated the areas GSB and GRC share 38 species, while GR1 and GR2 share 35 species (Fig. 2).

The Ordination analysis (Fig. 1) revealed two sharp groups of SU along axis I. The first group, on the right side of the graph, represents the areas with agricultural use (GSB and GRC) and shows higher levels of bare soil (BS). The second group, on the left, represents the areas used for extensive livestock grazing (GR1 and GR2). Some SUs of GR1, however, could be found at the right side of the graph. The two areas of more intensive use (GSB and GRC) were clearly separated along the axis II, in contrast to plots from the areas that were used only as pasture. A. affinis and P. pumilum were the species with the highest correlations to the first ordination axis. A. affinis was associated to areas previously used for agriculture (GSB and GRC), and P. pumilum to areas used only for livestock grazing (GR1 and GR2). The variable bare soil (BS) was most closely related to the GRC area $(\mathrm{p}<0.05)$.

The IVI values for bare soil (BS) were higher in GSB and GRC than in GR1 and GR2 (Tab. 3). A similar situation was also described by Garcia (2005) for areas with a history of rice crop (Tab. 4). The agricultural use 
Axis II (20.47\%)

$\mathrm{P}=0.3$

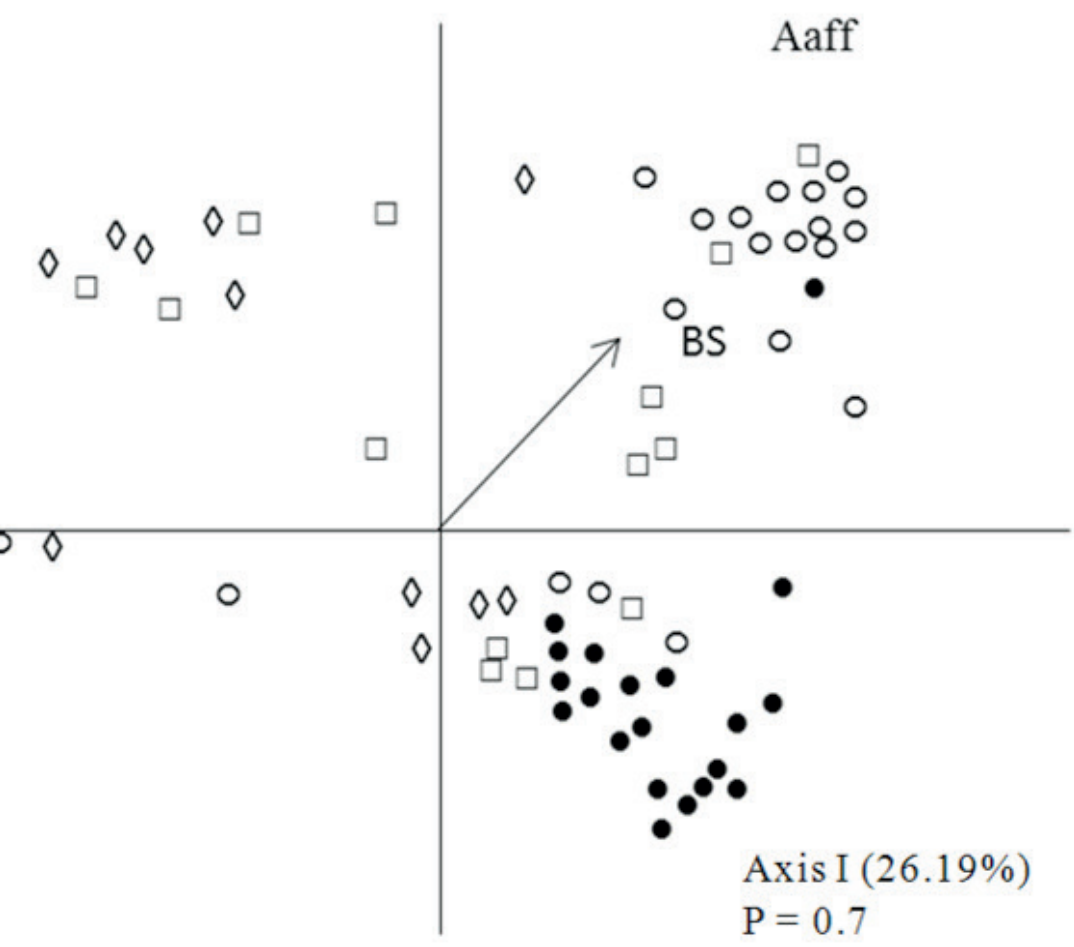

Fig. 1. Principal Coordinates Analysis of four grassland with different historical use, described for 21 variables and 80 SU. $\bullet=$ GSB, $\circ=$ GRC, $\square$ $=\mathrm{GR} 1, \diamond=\mathrm{GR} 2$, Aaff $=$ Axonopus affinis, Ppum $=$ Paspalum pumilum .

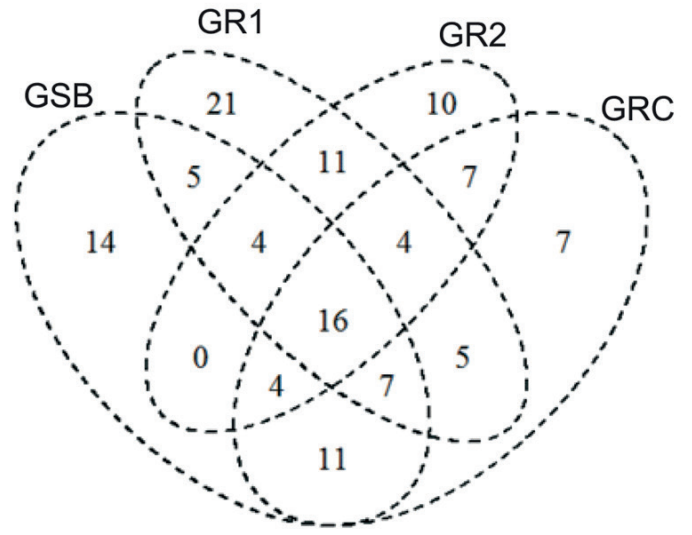

\begin{tabular}{|c|c|c|c|c|}
\hline & GSB & GRC & GR1 & GR2 \\
\hline GSB & 1 & & & \\
\hline GRC & 0.45 & 1 & & \\
\hline GR1 & 0.31 & 0.31 & 1 & \\
\hline GR2 & 0.26 & 0.36 & 0.37 & 1 \\
\hline
\end{tabular}

Fig. 2. Jaccard index showing similarity and plant species shared between the four grassland. São Lourenço do Sul, RS. GSB = grassland soybean/ ryegrass, GRC = grassland rice/livestock. GR1 = grassland extensive livestock breeding $1, \mathrm{GR} 2$ = grassland extensive livestock breeding 2 . is probably the factor influencing the increase of bare soil. The mid-season drainage of rice crops in Alfisols areas causes soil compaction (Streck et al. 2008) that limits the propagation of roots and the establishment of characteristic species of the region. In GR1 and GR2, high coverage of rhizomatous and stoloniferous species are favored by intensive grazing management (Tab. 3), and responsible for the low percentage of open soil.

Four species are in some degree endangered in the state of Rio Grande do Sul (SEMA 2014). In GRC, we recorded Eryngium divaricatum Hook. \& Arn and Lilaea scilloides A.W. Hill. Laurembergia tetrandra (Schott) Kanitz was recorded in GRC and in GR2. In GR1 and GR2 we recorded Tibouchina asperior (Cham.) Cogn. According to Species Link (CRIA 2015), E. divaricatum was recorded only three times in Brazil, the last record being was 30 year ago. Soliva macrocephala Cabrera has six records in Brazil (CRIA 2015), and seems to be restricted to Quaternary soils as an edaphic endemic taxon (Ferreira \& Boldrini 2011).

The presence of these endangered species indicates that the vegetation managed to return to a state containing some characteristic elements of native grassland vegetation of the region, evidencing some degree of resilience of the plant community, and thus that rotational land management or fallow cycles can contribute to biodiversity conservation. High species number in GSB may seem surprising at first. 
However, community composition and species abundance showed clear differences from natural grasslands, with dominance of ruderal and exotic species. The exotic species will probably remain in the vegetation for longer periods, which may hamper succession to native vegetation. In areas with current or past agricultural use (GSB and GRC), few native perennial species with high cover values were recorded (Tab. 3). The higher IVI of annual and exotic species is indicative of the degradation level of the areas. The high proportion of annuals in GSB, a species group with low importance in southern Brazilian grasslands (Overbeck \& Pfadenhauer 2007), also indicates that current vegetation is far from its original state and reflects recent or continuing disturbances.

Land-use choices by farmers are a consequence of abiotic characteristics. Grasslands on Entisols are not subject to land-use changes, because of the high erosion susceptibility of the soil. Alfisols, on the other hand, were covered by rice and soybean plantations. Our results show that the short-term use of agricultural areas as grassland, between crop cycles, can contribute, to some extent, to maintenance of grassland species, including some exotic ones. However, overall patterns of composition were distinct in these areas, and it was not the typical perennial species that dominated the two areas with a history of more intensive land use. Based on the features we found (proportion of exotics and ruderals), we can conclude that the grassland type that is established in the fallow does not present the typical features of coastal plain grasslands. From a conservation point of view, it seems necessary that parts of grassland vegetation on Alfisols would be preserved. The establishment of Legal Reserve areas and the necessity of licensing for suppression of native vegetation, according to the Brazilian Native Vegetation Protection Law (Brasil 2012), combined with an appropriate grassland systems management (i.e. grazing) could promote the conservation of grassland biodiversity and the sustainability of the agriculture on different soil types in the coastal region of southern Brazil.

\section{ACKNOWLEDGEMENTS}

We thank Silvia Guimarães de Souza for permission to work in the studied area. We thank the Coordenação de Aperfeiçoamento de Pessoal de Nível Superior (CAPES) for a PhD fellowship.

\section{REFERENCES}

Andrade, B.O., Koch, C., Boldrini, I.I., Vélez-Martin, E., Hasenack, H., Hermann, J.M., Kollmann, J., Pillar, V.D. \& Overbeck, G.E. 2015. Grassland degradation and restoration: a conceptual framework of stages and thresholds illustrated by southern Brazilian grasslands. Natureza \& Conservação 13(2):95-104.

Angiosperm Phylogeny Group IV. 2016. An update of the Angiosperm Phylogeny Group classification for the orders and families of flowering plants: Angiosperm Phylogeny Group IV. Botanical Journal of Linnean Society 181:1-20.

Bellemare, J., Motzkin, D., Foster, D.R. \& Forest, H. 2002. Legacies of the agricultural past in the forested present: an assessment of historical land-use effects on rich mesic forests. Journal of Biogeography 29:1401-1420.

Benton, T.G., Vickery, J. \& Wilson, J.D. 2003. Farmland biodiversity: is habitat heterogeneity the key? Trends in Ecology \& Evolution 18(4):182-188.

Boldrini, I.I., Trevisan, T. \& Schneider, A. 2008. Estudo florístico e fitossociológico de uma área às margens da lagoa do Armazém, Osório, Rio Grande do Sul, Brasil. Revista Brasileira de Biociências 6(4):355-367.

Boldrini, I.I. 2009. A flora dos campos do Rio Grande do Sul. In Campos Sulinos: conservação e uso sustentável da biodiversidade (V.D.P. Pillar, S.C. Müller, Z.M.S. Castilhos \& A.V.A. Jacques eds.). Ministério do Meio Ambiente, Brasília, p.63-77.

Boldrini, I.I. \& Longhi-Wagner, H.M. 2011. Poaceae no Rio Grande do Sul: diversidade, importância na fisionomia e conservação. Ciência e Ambiente - Botânica do Cone Sul 42:71-92.

Centro de Referência em Informação Ambiental. CRIA. 2015. Available at: http://splink.cria.org.br/. Accessed on 02/18/2015.

Cordeiro, J.L.P. \& Hasenack, H. 2009. Cobertura vegetal atual do Rio Grande do Sul. In Campos Sulinos: conservação e uso sustentável da biodiversidade. Brasília, Ministério do Meio Ambiente (V.D.P. Pillar, S.C. Müller, Z.M.S. Castilhos \& A.V.A. Jacques eds.). Ministério do Meio Ambiente, Brasília, p.285-299.

Coughneour, M.B. 1985. Graminoid responses to grazing by large herbivores adaptations, exaptations and interactions processes. Annual Missouri Botanical Garden 72:852-863.

Empresa Brasileira de Pesquisa Agropecuária. EMBRAPA. 1997. Manual de métodos de análise de solos. Centro Nacional de Pesquisa do Solo, Rio de Janeiro. 212p.

Empresa Brasileira de Pesquisa Agropecuária. EMBRAPA. 2014. Laboratório de Agrometeorologia. Dados meteorológicos on line. Available at: http://www.cpact.embrapa.br/agromet/. Accessed on $06 / 12 / 2014$.

Ferreira, P.M. \& Setubal, R.B. 2009. Florística e fitossociologia de um campo natural no município de Santo Antônio da Patrulha, Rio Grande do Sul, Brasil. Revista Brasileira de Biociências 7(2):195-204.

Ferreira, P.M. \& Boldrini, I.I. Potential Reflection of Distinct Ecological Units in Plants Endemism Categories. Conservation Biology 25(4):672-679.

Instituto de Botánica Darwinion. IBODA. Catálogo de las Plantas Vasculares del Cono Sur. Available at: http://www2.darwin.edu. ar/Proyectos/FloraArgentina/fa.htm. Accessed on 03/16/2015.

Fundação Instituto Brasileiro de Geografia e Estatística. IBGE. 2012. Manual técnico da vegetação brasileira. 2nd ed. Available at: ftp:// geoftp.ibge.gov.br. Accessed on 03/20/2016.

Instituto Rio Grandense do arroz. IRGA. 2013. Available at: http://www. irga.rs.gov.br/conteudo/4267/expointer:-irga-e-ccgl-tec-lancama-tecirga-6070rr . Accessed on 03/20/2015.

Garcia, E.N. 2005. Subsídios à conservação de campos no norte da Planície Costeira do Rio Grande do Sul. PhD thesis. 116f. Universidade Federal do Rio Grande do Sul. Rio Grande do Sul, Brasil.

Grime, J.P. 2002. Plant strategies, vegetation processes and ecosystem properties. John Wiley \& Sons, New York. 456p.

Harrison, S., Cornell, H. \& Moore, K. A. 2010. Spatial niches and coexistence: testing theory with tarweeds. Ecology 91(7):2141-2150.

Harrison, S. \& Emilio, B. 1999. Habitat fragmentation and large-scale conservation: what do we know for sure? Ecography 22(3):225-232.

Jackson, S.T. \& Sax, D.F. 2010. Balancing biodiversity in a changing environment: extinction debt immigration credit and species turnover. Trends in ecology \& evolution. 25(3):153-160.

Justus, G.O., Machado, M.L.A. \& Franco, M.S.M. 1986. Geomorfologia. In Projeto Radar da Amazônia, Brasil. RADAM Brasil. Levantamento de recursos naturais V. 53 Folha SH. 22 p.313-404. Porto Alegre e parte das folhas SH. 21 Uruguaiana e SI. 22 Lagoa Mirim. Instituto Brasileiro de Geografia e Estatística.

Li, H., Parent, LI., Karam, A. \& Tremblay, C. 2004. Potential of Sphagnum peat for improving soil organic matter, water holding capacity, bulk density and potato yield in a sandy soil. Plant and Soil. 265:355-365.

Londo, G., 1976. The decimal scale for releves of permanent quadrants. Vegetatio 33(1):61-64. 
Lopes, M.L.T., Carvalho, P.C.F., Anghiononi, I., Santos, D.T., Aguinaga, A.A.Q., Flores, J.P.C. \& Moraes, A. 2009. Sistema de integração lavoura-pecuária: efeito do manejo da altura em pastagem de aveia preta e azevém annual sobre o rendimento da cultura da soja. Ciência Rural 39(5):1499-1506.

Magurran, A.E. 2004. Measuring biological diversity. Blackwell, Malden $215 \mathrm{p}$.

Matteucci, S.D. \& Colma, A. 1982. Metodologia para el estudio de la vegetación. Secretaria General de la Organizacion de los Estados americanos. Programa Regional de Desarrollo Cientifico y Tecnológico, Washington, D. C. $168 \mathrm{p}$.

Mclauchlan, K. 2007. The nature and longevity of agricultural impacts on soil carbon and nutrients: a review. Ecosystems 9(8):1364-1382.

Medeiros, R.B., Favareto, R., Ferreira, O.C.L. \& Siewerdt, L., 2006. Persistência de Desmodium incanum DC. em meio a cultivos agrícolas estabelecidos sobre campo nativo. Pesquisa Agropecuária Gaúcha 12(1):37-44.

Oksanen, L. 2001. Logic of experiments in ecology: is pseudoreplication a pseudoissue? Oikos 94:27-38.

Overbeck, G.E. \& Pfadenhauer, J. 2007. Adaptive strategies in burned subtropical grassland in southern Brazil. Flora 202:27-49.

Overbeck, G.E., Muller, S.C., Fidelis, A., Pfadenhauer, J., Pillar, V.P., Blanco, C.C., Boldrini, I.I., Both, R. \& Forneck, E.D. 2007. Brazil's neglected Biome: the south Brazilian campos. Perspectives in Plant Ecology Evolution and Systematics 9:101-116.
Pillar, V.P. 2006. MULTIV. Software para análise multivariada, testes de aleatorização e autoreamostragem "bootstrap", v. 2.4.2. Porto Alegre: Departamento de Ecologia, UFRGS. Disponível em: http:// ecoqua.ecologia.ufrgs.br/MULTIV.html. Aceessed on 03/15/2015.

Santos, H.G., Jacomine, P.K.T., Anjos, L.H.C., Oliveira, V.O., Oliveira, J.B., Coelho, M.R., Lumbreras, J.F. \& Cunha, T.J.F. 2006. Sistema brasileiro de classificação dos solos. Empresa Brasileira de Pesquisa Agropecuária. 286p.

Secretaria do Meio Ambiente, 2014. Lista final das espécies da flora ameaçada - RS. Decreto Estadual n51.109. Disponível em: http:// www.fzb.rs.gov.br/conteudo/4809/?Homologada_a_nova Lista_da_Flora_Gaúcha_Ameaçada_de_Extinção. Accessed on $03 / 02 / 2015$.

Soil Survey Staff. 2014. Keys to soil taxonomy. United States Department of Agriculture. USDA. Natural Resources Conservation Service, Washington, DC. 369p.

Streck, E.V., Kämpf, N., Dalmolin, R.S.D., Klamt, E., Nascimento, P.C., Scneider, P., Giasson \& Pinto, L.F.S. 2008. Solos do Rio Grande do Sul. Porto Alegre: Empresa de Assistência Técnica e Extensão Rural. EMATER/RS. 222p.

Tilman, D., Cassman, K.G., Matson, P.A., Naylor, R. \& Polasky, S. 2002. Agricultural sustainability and intensive production practices. Nature 418:671-677. 